\title{
Inspection Allocation in Manufacturing Systems : A Genetic Algorithm Approach
}

\author{
Mukesh Taneja \\ N. Viswanadham * \\ Department of Computer Science \& Automation \\ Indian Institute of Science \\ Bangalore-560012, India
}

\begin{abstract}
In this paper, we are concerned with the problem of location of inspection centers in a multistage manufacturing system. A Genetic algorithm based approach is developed to determine the location of inspection centers resulting in a minimum expected total cost. The total cost includes inspection, manufacturing and scrapping cost at each stage of the manufacturing process. A penalty cost is included in it to account for a defective item which is not detected by the inspection scheme. A set of test problems are solved using this algorithm.
\end{abstract}

\section{Introduction}

In this paper, we design a Genetic Algorithm (GA) for locating the inspection stations in a multi-stage manufacturing system. A manufacturing system may be serial or nonserial. In a serial multi-stage manufacturing system, raw material is transformed into the final product in a series of discrete manufacturing stages. In a nonserial system certain manufacturing stages may involve joining the results of previous stages. If a manufacturing operation is not performed properly, some of the product units may become nonconforming. It is necessary to perform quality assurance inspections to prevent these nonconforming products from reaching the customer. However, the introduction of inspection centers into the manufacturing process entails additional costs and this results in an increase in the total cost. Careful analysis is required to determine which inspection centers are sufficiently useful to justify the additional cost.

Inspection allocation models are formulated with the objective of determining the number and location of inspection stations which will minimize the expected total cost per unit produced. The total cost includes some or all of the following : inspection cost, scrap cost, repair cost and penalty cost associated with shipping a nonconforming unit. Possible constraints on the above problem are based on an accepted outgoing quality level (AOQL) and/or on a limit on the maximum number of inspection centers that may be used.

Ignoring the possibility that inspection procedures could conceivably be integrated into the manufactur-

\footnotetext{
*Author to whom all the correspondence should be addressed, Email : vishu@bhaskara.csa.iisc.ernet.in
}

ing operation itself, $2^{N}$ possible inspection location alternatives exist for a $N$ stage manufacturing system. For all but the modest $N$, identification of the minimum cost inspection allocation plan by a complete enumeration of combinations becomes prohibitive. Many solution approaches have been employed to solve this problem with reasonable computational effort. The most common of these include dynamic programming ([2]), mixed integer programming $([5])$, simulation and heuristics based methods. The recursive optimization techniques, when applied to small manufacturing lines, yield the solution with reasonable computational effort. However, they become computationally expensive for large manufacturing lines (i.e., when $N$ is large). Also, it is difficult to apply recursive techniques if the optimization problem is constrained. Over the past few years, many new techniques have evolved to solve the hard problems arising in optimization. The two approaches which seem to be promising are Genetic Algorithms and Neural Networks.

We formulate a general inspection problem, both for a serial and a nonserial manufacturing system. We design a genetic algorithm to solve some special cases of the problem. It is shown that this problem maps fairly well into the representation required by a GA. The remainder of the paper is organized as follows. In section 2, we formulate the general inspection allocation problem. An overview of GAs is presented in section 3. Experimental results are presented in section 4 conclusions are drawn in section 5 .

\section{Problem Formulation}

\subsection{Serial Multi-Stage System}

A serial multi-stage manufacturing process is shown in figure 1 . There are $\mathrm{N}$ discrete manufacturing stages through which the work in process is routed in a fixed sequence. Each stage of the manufacturing process receives as input a batch or stream of identically processed items, which contains some mix of conforming and nonconforming items. The option is available to locate an inspection center before each manufacturing stage. If extra-high quality is required, repeated inspections may be performed. The objective is to find the number of repeated inspections at each stage such that the total cost of the manufacturing process is minimized. We make the following assumptions : 


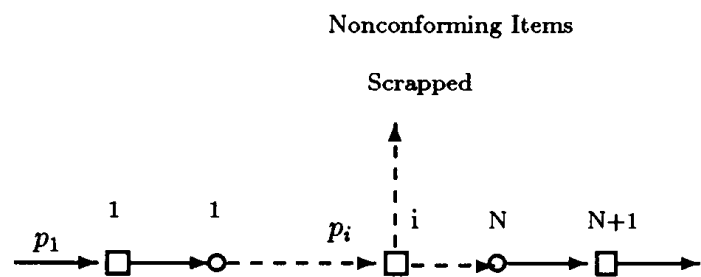

\section{Inspection Center \\ - Processing Stage}

Figure 1: Serial Manufacturing Process

1. The probability of being conforming is known for each item entering the manufacturing line.

2. An inspection operation may involve errors of two types. It may reject a conforming item or accept a nonconforming item. These are referred to as type I and type II errors respectively. The probabilities of these errors are known at each stage. Also, these are constant for each inspection level at a particular stage. Here, an inspection level for a stage refers to the number of repeated inspections performed at that stage.

3. All the nonconforming items are scrapped.

4. The inspection costs, scrapping costs and manufacturing costs are known for each stage in the manufacturing line.

5. If a nonconforming item reaches the customer, a penalty cost is incurred. The penalty cost is taken into account after the last stage in figure 1.

In order to specify the cost elements, we consider the system in figure 1 with repeated inspections at each stage of the manufacturing system. If an item is conforming and is accepted at all the repeated inspections, then the total cost would be equal to the cost of inspection plus the cost of manufacturing that item. If the item is nonconforming and is not rejected at any of the repeated inspections, the total cost would be equal to the sum of the inspection cost, penalty cost and the manufacturing cost. If an item is rejected at some inspection station, then the total cost would be equal to the sum of the inspection cost, scrapping cost and manufacturing cost.

Let $p_{i}$ be the probability that any single item is conforming at stage $i$. If we choose to inspect at stage $i$, an inspection cost of $n_{i}$ occurs for each item inspected. Here, one or more measurements (for testing) are taken for an item and a decision has to be made whether to accept or reject the item. The accuracy of these measurements determines the prevalence of type I (false reject) and type II (false accept) errors. Let $\alpha_{i}$ be the probability of type I error. Thus if an item is conforming, it will be rejected with probability $\alpha_{i}$ during an inspection process at stage $i$. Similarly, let $\beta_{i}$ be the probability of type II error i.e. a nonconforming item will be accepted with probability $\beta_{i}$. The number of repeated inspections performed at stage $i$ is denoted by $x_{i}$. The probability of accepting an item after $x_{i}$ inspections at stage $i$ is denoted by $A_{i}\left(p_{i}, x_{i}\right)$. Then,

$$
A_{i}\left(p_{i}, x_{i}\right)=A_{i-1}\left(p_{i-1}, x_{i-1}\right) \tilde{A}_{i}\left(p_{i}, x_{i}\right)
$$

Here,

$$
\tilde{A}_{i}\left(p_{i}, x_{i}\right)=p_{i}\left(1-\alpha_{i}\right)^{x_{i}}+\left(1-p_{i}\right) \beta_{i}^{x_{i}}
$$

Let $s_{i}$ be the scrapping cost at stage $i$ respectively. The scrapping cost represents the income generated by selling nonconforming units as scrap or lower grade products. This is treated as a negative cost. The unit manufacturing cost of an item at stage $i$ is denoted by $c_{i}$. A manufacturing stage may cause nonconformity in an item during the manufacturing. The probability that a conforming item becomes nonconforming through stage $i$ is denoted by $f_{i}$. The objective function to be minimized at the $i$ th stage is the expected total cost, given that the input has probability $p_{i}$ of being conforming. The total expected $\operatorname{cost} T_{i}\left(p_{i}, x_{i}\right)$ is equal to the manufacturing cost at stage $i$ when $x_{i}=0$. If $x_{i}>0$ then $T_{i}\left(p_{i}, x_{i}\right)$ is the sum of three types of costs : inspection cost, scrapping cost and manufacturing cost. The expected inspection cost at the $i$ th stage is given by :

$$
I_{i}\left(p_{i}, x_{i}\right)=A_{i-1}\left(p_{i-1}, x_{i-1}\right) N_{i}\left(p_{i}, x_{i}\right)
$$

Here, $N_{i}\left(p_{i}, x_{i}\right)$ is given by :

$$
\begin{aligned}
N_{i}\left(p_{i}, x_{i}\right)=\quad n_{i} x_{i}\left(1-p_{i}\right) \beta_{i}^{x_{i}}+n_{i} x_{i} p_{i}\left(1-\alpha_{i}\right)^{x_{i}} \\
+n_{i}\left(1-p_{i}\right) \sum_{k=1}^{x_{i}} k\left(1-\beta_{i}\right) \beta_{i}^{k-1} \\
+n_{i} p_{i} \sum_{k=1}^{x_{i}} k \alpha_{i}\left(1-\alpha_{i}\right)^{k-1}
\end{aligned}
$$

Here, the four terms correspond to undetected nonconforming item, accepted conforming item, detected nonconforming item and rejected conforming item respectively. The summation in the second term represents the expected number of inspections for rejection of nonconforming item. The summation in the fourth term represents the expected number of inspections for the rejection of conforming items. Equation (4) is valid for $1 \leq i \leq N+1$. If $\alpha_{i} \neq 0$ and $\beta_{i} \neq 1$, then equation (4) can be simplified to :

$N_{i}\left(p_{i}, x_{i}\right)=\frac{n_{i} p_{i}\left(1-\left(1-\alpha_{i}\right)^{x_{i}}\right)}{\alpha_{i}}+\frac{n_{i}\left(1-p_{i}\right)\left(1-\beta_{i}^{x_{i}}\right)}{1-\beta_{i}}$

The probability that an item is rejected is given by :

$$
\tilde{D}_{i}\left(p_{i}, x_{i}\right)=p_{i} \sum_{k=1}^{x_{i}} \alpha_{i}\left(1-\alpha_{i}\right)^{k-1}+\left(1-p_{i}\right) \sum_{k=1}^{x_{i}}\left(1-\beta_{i}\right) \beta_{i}^{k-1}
$$


The above equation (6) can be simplified to :

$$
\tilde{D}_{i}\left(p_{i}, x_{i}\right)=p_{i}\left(1-\left(1-\alpha_{i}\right)^{x_{i}}\right)+\left(1-p_{i}\right)\left(1-\beta_{i}^{x_{i}}\right)
$$

Let $M_{i}\left(p_{i}, x_{i}\right)$ be the modified probability that an item is conforming after $x_{i}$ inspections. Then,

$$
M_{i}\left(p_{i}, x_{i}\right)=\frac{p_{i}\left(1-\alpha_{i}\right)^{x_{i}}}{\tilde{A}_{i}\left(p_{i}, x_{i}\right)}
$$

The expected scrapping cost is given by :

$$
S_{i}\left(p_{i}, x_{i}\right)=s_{i} A_{i-1}\left(p_{i-1}, x_{i-1}\right) \tilde{D}_{i}\left(p_{i}, x_{i}\right)
$$

The manufacturing cost at the $i$ th stage is given by :

$$
P_{i}\left(p_{i}, x_{i}\right)=c_{i} A_{i}\left(p_{i}, x_{i}\right)
$$

The equations (1) to (10) are valid for $x_{i}>0$. The total cost incurred at the $i$ th stage is given by :

$$
T_{i}\left(p_{i}, x_{i}\right)= \begin{cases}P_{i}\left(p_{i}, x_{i}\right) & \text { if } x_{i}=0 \\ I_{i}\left(p_{i}, x_{i}\right)+S_{i}\left(p_{i}, x_{i}\right) & \text { if } x_{i}>0 \\ +P_{i}\left(p_{i}, x_{i}\right) & \end{cases}
$$

The probability of the output stream of the $i$ th stage is given by :

$$
p_{i+1}= \begin{cases}p_{i}\left(1-f_{i}\right) & \text { if } x_{i}=0 \\ M_{i}\left(p_{i}, x_{i}\right)\left(1-f_{i}\right) & \text { if } x_{i}>0\end{cases}
$$

Once the manufacturing process is completed, all items are supplied to the customers. Let $d$ be the cost of shipping a defective item to a customer site. It includes cost of a field repair, cost of the analysis \& repair of the defective item that comes back to the plant and a cost measuring the customer's loss of good will. It represents the penalty cost occurred in the whole manufacturing process. The revenue obtained from selling an item is denoted by $v$. The net income generated by selling that item is given by :

$$
G\left(p_{N+2}\right)=A_{N+2}\left[p_{N+2}(d+v)-d\right]
$$

Let the vector $\bar{x}=\left(x_{1}, \cdots, x_{N+1}\right)^{T}$ specify the decisions taken in the manufacturing line. It is to be noted that $x_{i}$ can take only nonnegative integer values. Given the input probability $p_{1}$ and the decision vector $\bar{x}$, the total cost can be computed as :

$$
T\left(p_{1}, \bar{x}\right)=\sum_{i=1}^{i=N+1} T_{i}\left(p_{i}, x_{i}\right)-G\left(p_{N+2}\right)
$$

Here, $T_{i}$ and $G\left(p_{N+2}\right)$ are defined in equations (11) and (13) respectively. The objective is to find a vector $\bar{x}$ such that the total cost in equation 14 is minimized for a given $p_{1}$. Thus the above optimization problem is defined as :

$$
\begin{aligned}
\mathrm{P} 1: & \min T\left(p_{1}, \bar{x}\right) \\
& \text { subject to } x_{i} \geq 0 \quad \forall i \in[1, N+1]
\end{aligned}
$$

Depending on the nature of process technology and economic limitations, it may be necessary to incorporate some additional constraints into the above formulation. These constraints can be of the following types :

1. Maximum number of inspection centers that can be used.

2. Minimum acceptable outgoing quality level required.

3. Maximum number of repeated inspections allowed at each stage.

If all of the above constraints are present, then the constrained problem becomes :

$$
\begin{aligned}
\mathrm{P} 2: & \min T\left(p_{1}, \bar{x}\right) \\
\text { subject to } L & \leq L_{\max } \\
& p_{N+2} \geq p^{*} \\
& 0 \leq x_{i} \leq z_{i}
\end{aligned}
$$

Here, $L$ is the actual number of inspection centers located and $L_{\max }$ is the maximum number of inspection centers that can be used. The acceptable outgoing quality level (AOQL) is denoted by $p^{*}$. The vector $\bar{z}=\left[z_{1}, \cdots, z_{N+1}\right]$ denotes the maximum number of repetitive inspections allowed.

Similarly, if we assume that all the rejected items are reworked, then the corresponding equations are listed in (17). Here we assume that a reworked item is always good and it reenters the manufacturing line.

$$
\begin{aligned}
I_{i}\left(p_{i}, x_{i}\right) & =N_{i}\left(p_{i}, x_{i}\right) \\
M_{i}\left(p_{i}, x_{i}\right) & =1-\left(1-p_{i}\right) \beta_{i}^{x_{i}} \\
R_{i}\left(p_{i}, x_{i}\right) & =r_{i} \tilde{D}_{i}\left(p_{i}, x_{i}\right) \\
P_{i}\left(p_{i}, x_{i}\right) & =c_{i}
\end{aligned}
$$

Here, $r_{i}$ denotes the reworking cost at stage $i$ and $R_{i}\left(p_{i}, x_{i}\right)$ denotes the expected reworking cost. Now, we discuss some special cases of the above problem.

\subsubsection{Case 1}

Assumptions :

1. Repetitive inspections are not allowed. Thus we can inspect only once at a stage.

2. All the rejected items are scrapped.

Thus,

$$
\begin{array}{ccccc}
x_{i}= & 0 & \text { or } & 1 & \forall i \in[1, N+1] \\
r_{i}= & 0 & & \forall i \in[1, N+1]
\end{array}
$$

In this case, a presence of an inspection center at stage $i$ is represented by $x_{i}=1$ and an absence is represented by $x_{i}=0$. The equations (2), (4), (7), (8) and 
(9) reduce to the following :

$$
\begin{aligned}
\tilde{A}_{i}\left(p_{i}\right) & =p_{i}\left(1-\alpha_{i}\right)+\left(1-p_{i}\right) \beta_{i} \\
N_{i}\left(p_{i}\right) & =n_{i} \\
\tilde{D}_{i}\left(p_{i}\right) & =p_{i} \alpha_{i}+\left(1-p_{i}\right)\left(1-\beta_{i}\right) \\
M_{i}\left(p_{i}\right) & =\frac{p_{i}\left(1-\alpha_{i}\right)}{\tilde{A}_{i}\left(p_{i}\right)} \\
S_{i}\left(p_{i}\right) & =s_{i} A_{i-1}\left(p_{i-1}\right) \tilde{D}_{i}\left(p_{i}\right)
\end{aligned}
$$

The cost incurred at the $i$ th stage is given by :

$$
T_{i}\left(p_{i}, x_{i}\right)= \begin{cases}P_{i}\left(p_{i}\right) & \text { if } x_{i}=0 \\ I_{i}\left(p_{i}\right)+S_{i}\left(p_{i}\right)+P_{i}\left(p_{i}\right) & \text { if } x_{i}=1\end{cases}
$$

The probability of the output stream of the $i$ th stage is given by :

$$
p_{i+1}= \begin{cases}p_{i}\left(1-f_{i}\right) & \text { if } x_{i}=0 \\ M_{i}\left(p_{i}\right)\left(1-f_{i}\right) & \text { if } x_{i}=1\end{cases}
$$

The above two expressions can be rewritten as :

$$
\begin{array}{cr}
T_{i}\left(p_{i}, x_{i}\right)=P_{i}\left(p_{i}\right)\left(1-x_{i}\right)+\left[I_{i}\left(p_{i}\right)+S_{i}\left(p_{i}\right)+P_{i}\left(p_{i}\right)\right] x_{i} \\
p_{i+1}=p_{i}\left(1-f_{i}\right)\left(1-x_{i}\right)+M_{i}\left(p_{i}\right)\left(1-f_{i}\right) x_{i}
\end{array}
$$

Eppen and Hurst ([2]) have discussed the unconstrained problem ( $\mathrm{P} 1)$, under the above assumptions. They formulate a recursive objective function and solve that using dynamic programming.

\subsubsection{Case 2}

Assumptions :

1. Repetitive inspections are not allowed.

2. All the rejected items are reworked.

Thus,

$$
\begin{array}{ccccc}
x_{i}= & 0 & \text { or } & 1 & \forall i \in[1, N+1] \\
s_{i}= & 0 & & \forall i \in[1, N+1]
\end{array}
$$

Corresponding equations are given by :

$$
\begin{aligned}
I_{i}\left(p_{i}\right) & =n_{i} \\
R_{i}\left(p_{i}\right) & =r_{i} \tilde{D}_{i}\left(p_{i}\right) \\
S_{i}\left(p_{i}\right) & =0
\end{aligned}
$$

The cost incurred at the $i$ th stage is given by :

$$
T_{i}\left(p_{i}, x_{i}\right)= \begin{cases}P_{i}\left(p_{i}\right) & \text { if } x_{i}=0 \\ I_{i}\left(p_{i}\right)+R_{i}\left(p_{i}\right)+P_{i}\left(p_{i}\right) & \text { if } x_{i}=1\end{cases}
$$

\subsubsection{Case 3}

Assumptions :

1. Repetitive inspections are allowed.

2. All the rejected items are scrapped.

3. Probability of type I error is zero.

Thus,

$$
\begin{array}{ccc}
r_{i}= & 0 & \forall i \in[1, N+1] \\
\alpha_{i}= & 0 & \forall i \in[1, N+1]
\end{array}
$$

Now, the equations (2), (7) and (8) reduce to the following :

$$
\begin{aligned}
\tilde{A}_{i}\left(p_{i}, x_{i}\right) & =p_{i}+\left(1-p_{i}\right) \beta_{i}^{x_{i}} \\
\tilde{D}_{i}\left(p_{i}, x_{i}\right) & =\left(1-p_{i}\right)\left(1-\beta_{i}^{x_{i}}\right) \\
M_{i}\left(p_{i}, x_{i}\right) & =\frac{p_{i}}{\tilde{A}_{i}\left(p_{i}, x_{i}\right)}
\end{aligned}
$$

The cost incurred at the $i$ th stage is given by :

$$
T_{i}\left(p_{i}, x_{i}\right)= \begin{cases}P_{i}\left(p_{i}, x_{i}\right) & \text { if } x_{i}=0 \\ I_{i}\left(p_{i}, x_{i}\right)+S_{i}\left(p_{i}, x_{i}\right) & \text { if } x_{i}>0 \\ +P_{i}\left(p_{i}, x_{i}\right) & \end{cases}
$$

\subsection{Nonserial Multi-Stage System}

We consider a special class of nonserial manufacturing system shown in figure 2. The inspection allocation problem for this configuration has been solved using dynamic programming in Garcia-Diaz [3]. We solve this problem using genetic algorithm. At each stage in figure 2, there are exactly two inputs. A basic module of this configuration is shown in figure 3 . The inspection centers at the upper and lower branch of stage $i$ are denoted by $i u$ and $i d$ respectively.

Notations :

$r_{i u}\left(r_{i d}\right) \quad$ rework cost at inspection station $i u(i d)$

$n_{i u}\left(n_{i d}\right) \quad$ inspection cost at inspection station $i u(i d)$

$p_{i u}\left(p_{i d}\right) \quad \mathrm{p}$ (conforming) at the input of $i u(i d)$

$p_{i u}^{\prime}\left(p_{i d}^{\prime}\right) \quad \mathrm{p}$ (conforming) at the output of $i u(i d)$

$p_{(i+1) u} \quad \mathrm{p}$ (conforming) at the output of stage $i$

$x_{i u}\left(x_{i d}\right) \quad$ number of repeated inspections at $i u(i d)$

$\alpha_{i u}\left(\alpha_{i d}\right) \quad \mathrm{p}$ (type I error) at iu (id)

$\beta_{i u}\left(\beta_{i d}\right) \quad \mathrm{p}($ type II error) at $i u(i d)$

$I_{i u}\left(I_{i d}\right) \quad$ expected inspection cost at $i u(i d)$

$R_{i u}\left(R_{i d}\right) \quad$ expected reworking cost at $i u(i d)$

$P_{i}$ assembly cost at stage $i$

We assume that all the rejected items are reworked. The inspection cost, reworking cost, manufacturing cost and the modified probability after inspection are given by equation (17) with subscript $i$ changed to $i u$ and $i d$ for the two inspection centers in figure 3 . The probability after inspection $\left(p_{i \mu}^{\prime}\right)$ at center $i u$ is equal to $M_{i u}$. If there is no inspection, then it is same as 


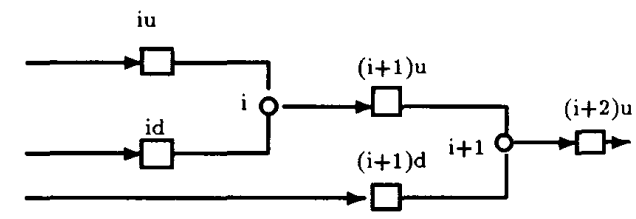

Inspection center

- Assembly Stage

Figure 2: Nonserial Manufacturing Process

$p_{i u}$ only. The probability of an item being conforming at the output is given by :

$$
\begin{aligned}
p_{(i+1) u}= & \left(1-f_{i}\right)+p_{i u}^{\prime}+p_{i d}^{\prime} \\
- & \left(1-f_{i}\right) p_{i u}^{\prime}-\left(1-f_{i}\right) p_{i d}^{\prime} \\
& -p_{i u}^{\prime} p_{i d}^{\prime}+p_{i u}^{\prime} p_{i d}^{\prime}\left(1-f_{i}\right)
\end{aligned}
$$

The total cost for the $i$ th basic module is given by :

$$
T_{i}=I_{i u}+I_{i d}+R_{i u}+R_{i d}+P_{i}
$$

Here, one or more of the above terms will be equal to zero, in case an item is not inspected at a stage. The total cost for a $N$ stage system is given by :

$$
T=\sum_{i=1}^{i=N+1} T_{i}-G\left(p_{N+2}\right)
$$

Here, we assume that the $(N+1)$ th stage act as a dummy stage with $I_{(N+1) d}=R_{(N+1) d}=P_{(N+1)}=$ 0 and $G\left(p_{N+2}\right)$ is as defined in equation (13). The output probability of this stage is given by :

$$
\begin{aligned}
p_{(N+2)}= & p_{(N+1) u}^{\prime}+\left(1-f_{(N+1)}\right) \\
& -p_{(N+1) u}^{\prime}\left(1-f_{(N+1)}\right)
\end{aligned}
$$

The optimization problem is to minimize the total cost given in equation (32). The next section presents a brief overview of genetic algorithms.

\section{Genetic Algorithms}

Genetic Algorithms (GAs) require the natural parameter set of the optimization problem to be coded as a finite-length string over some finite alphabet. The parameters in our implementation are coded as strings of 0 's and 1's. In the case, when only a single inspection is allowed, each parameter $x_{i}$ is represented by a one bit string. A ' 0 ' indicates absence of an inspection station at the $i$ th stage and a ' 1 ' indicates presence of an inspection center. Thus a solution is represented by a $(N+1)$ bit string $x_{1}, \cdots, x_{N+1}$, where each $x_{i}$ can take value either 0 or 1 . When repeated inspections

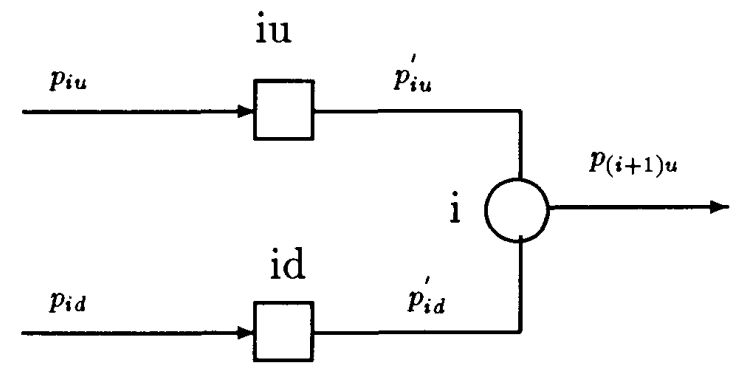

Figure 3: Basic Module of Nonserial Process

are allowed, each of these parameters can be coded as a finite length string of 0 's and 1's.

A simple GA is composed of the three operators : remanufacturing, crossover and mutation. These operators are applied to successive population to generate new population. A suitable combination of these parameters has to be found for the GA to work well on a given problem. Application of GAs to constrained problems require some special methods. We use the Exterior penalty method ([4]), which has been used successively in a number of problems. With this method, whenever a constraint is violated, the unconstrained objective function value is penalized by an amount related to a function of the constraint violation. The next section presents the results obtained by applying the GA on a set of test problems.

\section{Experimental Study}

The GA in its standard form assumes that the problem is a maximization problem and the objective function takes only positive values on its domain. Thus minimization problem in equation (15) is converted to the following maximization form :

$$
\begin{aligned}
\mathrm{P}^{\prime}: & \max T^{\prime}\left(p_{1}, \bar{x}\right) \\
& \text { subject to } x_{i} \geq 0 \quad \forall i \in[1, N+1]
\end{aligned}
$$

Here, $T^{\prime}=-T+C$ and $C$ is a large constant such that $T^{\prime}$ remains positive on its domain. Throughout our implementation, we use the Elitist expected value strategy for implementation of the remanufacturing operator of the GA. The crossover and mutation rates for the GA are taken to be 0.70 and 0.015 respectively.

We consider the single inspection case where it is possible to inspect at the most once at a stage. Thus $x_{i}$ can take value either 0 or 1 . Each solution in the GA is represented by a $(N+1)$ bit string $x_{1}, \cdots, x_{N+1}$ For example, a solution string 0101 means to place inspection centers after the first and the third manufacturing stage in a three stage system (fourth stage acts as a dummy stage). As the constraint in problem $P 1^{\prime}$ is automatically taken into account in the structure of a solution string of $\mathrm{GA}$, this problem is converted to 


\begin{tabular}{|l|r|r|r|r|r|r|}
\hline$i$ & $1-f_{i}$ & $\alpha_{i}$ & $\beta_{i}$ & $n_{i}$ & $s_{i}$ & $c_{i}$ \\
\hline 1 & 0.998 & 0.01 & 0.04 & 0.2 & -1 & 4 \\
2 & 0.985 & 0.01 & 0.04 & 0.5 & -1 & 5 \\
3 & 0.989 & 0.02 & 0.06 & 0.6 & -3 & 20 \\
4 & 0.992 & 0.03 & 0.07 & 0.8 & -5 & 8 \\
5 & 0.987 & 0.03 & 0.08 & 1.0 & -8 & 5 \\
6 & 1.000 & 0.04 & 0.08 & 1.1 & -12 & 0 \\
\hline
\end{tabular}

Table 1: Test Problem Statistics

\begin{tabular}{|l|c|}
\hline$p_{1}$ & Inspection Policy \\
\hline 0.40 & 110000 \\
0.60 & 101000 \\
0.70 & 101000 \\
0.80 & 100000 \\
0.90 & 100000 \\
0.95 & 100000 \\
\hline
\end{tabular}

Table 2: Inspection Allocation Policy for P1

the following unconstrained problem :

$$
\mathrm{P}^{u}{ }^{u}: \max \quad T^{\prime}\left(p_{1}, \bar{x}\right)
$$

Cases $1 \& 2$ of section 2.1 fall into this class of problems. To demonstrate characteristics of the solution, a five stage problem has been solved. The data for this problem is given in table 1 . A sixth stage is added to this table which acts as a dummy stage. The values for $d$ and $v$ are assumed to be 50 and 125 respectively. The experimental results are presented for the case 1 of section 2.1. The inspection policies obtained at different values of the incoming probabilities are shown in table 2. Table 3 gives the performance statistics of GA on a set of five test problems.

As mentioned earlier, we have used the penalty methods to solve the constrained problems. The constrained problem (P2) in our case has inequality constraints. Whenever a candidate solution violates a constraint, the corresponding objective function is penalized by an amount related to a function of the constraint violation. In other words, a constrained problem is transformed to an unconstrained problem by associating a penalty with all constraint violations and the penalties are included in the function evaluation.

\begin{tabular}{|l|c|c|c|}
\hline $\begin{array}{l}\text { Problem } \\
\text { No. }\end{array}$ & $\begin{array}{c}\text { No. of } \\
\text { Stages }\end{array}$ & $\begin{array}{c}\text { Population } \\
\text { Size }\end{array}$ & $\begin{array}{c}\text { Number of } \\
\text { Generations }\end{array}$ \\
\hline 1 & 6 & 4 & 9 \\
2 & 10 & 6 & 72 \\
3 & 16 & 8 & 186 \\
4 & 20 & 8 & 568 \\
5 & 30 & 10 & 1020 \\
\hline
\end{tabular}

Table 3: Performance of GA on a test set for P1

\begin{tabular}{|l|c|}
\hline$p_{1}$ & Inspection Policy \\
\hline 0.60 & 100000 \\
0.70 & 100000 \\
0.80 & 100000 \\
0.90 & 100000 \\
0.95 & 100000 \\
\hline
\end{tabular}

Table 4: Inspection Allocation Policy with constraint on $L_{\max }$

However, though the function evaluation is well defined, there is no accepted methodology for combining it with the penalty. Davis [1] discusses this problem listing disadvantages of using high, moderate or light penalties. In our implementation, we square the violation of constraint. For example in P2, whenever we find that $L>L_{\max }$, we penalize the objective function by an amount proportional to $\left(L-L_{\max }\right)^{2}$, i.e. we evaluate the following :

$$
T^{\prime}-P *\left(L-L_{\max }\right)^{2}
$$

Here, $P$ is a positive constant. Rest of the algorithm proceeds as usual. Same technique is used to deal with other constraints. We consider the earlier problem (table 1) again. The inspection allocation policies generated for the unconstrained problem P1 were given in table 2 . Now, we restrict the number of maximum inspection centers that can be located to one (i.e. $\left.L_{\max }=1\right)$. The new inspection policies are presented in table 4.

\section{Conclusions}

We have formulated an inspection allocation problem for a multistage manufacturing system where repeated inspections are allowed. We have designed a genetic algorithm to solve this problem. Experimental results are presented for some special cases of this formulation on a set of test problems.

\section{References}

[1] L. Davis, (Editor), Genetic Algorithms and Simulated Annealing, Pitman, London, 1987.

[2] G. D. Eppen, \& E. G. Hurst, "Optimal Location of Inspection Stations in a Multi-stage Production Process", Management Science, 20, 1974.

[3] A. Garcia-Diaz, J. W. Foster, \& M. Bonyuet, "Dynamic Programming Analysis of Multi-Stage Inspection Systems", IIE Transactions, June, 1984.

[4] D. E. Goldberg, Genetic Algorithms in Search, Optimization and Machine Learning, AddisonWesley, 1989.

[5] B. Yum, \& E. D. McDowell, "Optimal Inspection Policies in a Serial Production System Including Scrap Rework and Repair : An MILP Approach", Int. Journal of Production Research, Vol 25, No. 10, 1451-1464, 1987. 